\title{
Fundamentals of Mobility in Cellular Networks: Modeling and Analysis
}

\author{
Xingqin Lin, Radha Krishna Ganti, Philip J. Fleming and Jeffrey G. Andrews
}

\begin{abstract}
Mobility modeling and analysis is a key issue in wireless networks, but there are surprisingly few fundamental results. In this paper we propose a new and quite general random waypoint (RWP) mobility model which is valid over the entire plane. We derive key properties of the proposed mobility model including transition length, transition time and spatial node distribution. Then the RWP mobility model is applied to study the handover rate in cellular networks under both deterministic (hexagonal) and random (Poisson) base station (BS) models. Closed form expressions for handover rate can be obtained, most of which to our knowledge are the first of their kind. For example, these results show the expected property that the handover rate is proportional to the square root of base station density. Also, we find that Poisson-Voronoi model for BS coverage areas is about as accurate in terms of mobility (particularly handover) evaluation as the ubiquitous hexagonal model.
\end{abstract}

\section{INTRODUCTION}

The support of mobility is a fundamental aspect of wireless networks [1], [2]. Mobility management is taking on new importance and complexity in emerging cellular networks, which have ever-smaller and more irregular cells. Mobility has generally been handled heuristically, and indeed there are very few analytic results or even widely accepted models on the fundamentals of mobility, e.g. the handover rate as a function of speed in a cellular network.

One approach is to construct mobility models based on empirical mobility patterns, known as trace models [1]. Though considered accurate, they require the knowledge of many detailed network characteristics and rule out general analytical models and results. Therefore, so-called synthetic models are often utilized to analyze the impact of mobility on network performance and to evaluate protocols in simulation. Popular synthetic models include random walk, random waypoint (RWP), and Gauss-Markov [1].

In this paper, we focus on RWP mobility model originally proposed in [3]. In this model, mobile users move in a finite domain $\mathcal{A}$. At each turning point, the mobile user selects the destination point (referred to as waypoint) uniformly distributed in $\mathcal{A}$ and chooses the velocity from some distribution. Then the user moves along the line connecting its starting waypoint to the newly selected waypoint at the chosen

Xingqin Lin and Jeffrey G. Andrews are with Department of Electrical \& Computer Engineering, The University of Texas at Austin, USA. (E-mail: xlin@utexas.edu, jandrews@ece.utexas.edu). Radha Krishna Ganti is with Department of Electrical Engineering, Indian Institute of Technology, Madras, India. (E-mail: rganti@ee.iitm.ac.in). Philip J. Fleming is with Nokia Siemens Networks (E-mail: phil.fleming@nsn.com). This research was supported by Nokia Siemens Networks. velocity. This process repeats at each waypoint. Optionally, the mobile user can have a random pause time at each waypoint before moving to the next waypoint. A drawback of the RWP mobility model is that the stationary spatial node distribution tends to concentrate near the centre of the finite domain [4]. Thus, we propose a new RWP mobility model defined on the entire plane and then derive its associated properties.

We then apply this new RWP model to study handover rate in cellular networks whose BSs are modeled in two opposite ways. First is the traditional hexagonal grid, which represents an extreme in terms of regularity and is completely deterministic. The second is to model the BS locations as drawn from a Poisson point process (PPP), which creates a set of BSs with completely independent locations [5], [6]. Thus, most any real-world deployment would seem to fall in between these two extremes, since real BS deployments are neither perfectly regular nor independent. Under the PPP BS model, the cellular network can be viewed as a PoissonVoronoi tessellation if the mobile users are assumed to connect to the nearest BSs. Closed form expressions are obtained for handover rate under both models. To the best of our knowledge, most of these expressions are new.

The analysis in this paper requires stochastic geometric tools, which are becoming increasingly sophisticated and popular [7]-[10]. As far as mobility is concerned, [11] proposed a framework to study the impact of mobility in cellular networks modeled as Poisson-Voronoi tessellation. In particular, the authors proposed Poisson line process to model the road system, along which the mobile users move. Thus, the mobility pattern in [11] is of large scale while the RWP mobility model in this paper is of small scale. Our study can be viewed as a complementary one to [11] and gives valuable insights. For example, our result indicates that if cells decrease in size such that the BS density per unit area is increased by 4 times, then the handover rate would be doubled. Also, recent work showed that the PPP model for BSs was about as accurate in terms of SINR distribution as the hexagonal grid for a representative urban cellular network [12]. Interestingly, we find that this observation is also true for mobility evaluation, though the Poisson-Voronoi model yields slightly higher handover rate than hexagonal model.

Before ending this section, we remark that most of the proofs in this paper are omitted for brevity. For interested readers, we refer to [13]. 


\section{PRoposed RWP MOBILITY MODEL}

We describe the proposed RWP mobility model in this section. As in the description of traditional RWP model (see, e.g., [4]), the movement trace of a node can be formally described by an infinite sequence of quadruples: $\left\{\left(\boldsymbol{X}_{n-1}, \boldsymbol{X}_{n}, V_{n}, S_{n}\right)\right\}_{n \in \mathbb{N}}$, where $n$ denotes the $n$-th movement period. During the $n$-th movement period, $\boldsymbol{X}_{n-1}$ denotes the starting waypoint, $\boldsymbol{X}_{n}$ denotes the target waypoint, $V_{n}$ denotes the velocity, and $S_{n}$ denotes the pause time at the waypoint $\boldsymbol{X}_{n}$.

We next describe the way of selecting waypoints in $\mathbb{R}^{2}$. Given the current waypoint $\boldsymbol{X}_{n-1}$, the next waypoint $\boldsymbol{X}_{n}$ is chosen such that the included angle between the vector $\boldsymbol{X}_{n}-$ $\boldsymbol{X}_{n-1}$ and the abscissa is uniformly distributed on $[0,2 \pi]$ and the transition length $L_{n}=\left\|\boldsymbol{X}_{n}-\boldsymbol{X}_{n-1}\right\|$ is a nonnegative random variable. This selection of waypoints is independent and identical for each movement period.

Though there is a degree of freedom in modeling the random transition lengths, we focus on a particular choice in this paper. Specifically, the transition lengths $\left\{L_{1}, L_{2}, \ldots\right\}$ are chosen to be independent and identically distributed (i.i.d.) with cumulative distribution function (cdf)

$$
P(L \leq l)=1-\exp \left(-\lambda \pi l^{2}\right), l \geq 0
$$

i.e., the transition lengths are Rayleigh distributed. This selection bears an interesting interpretation: Given the waypoint $\boldsymbol{X}_{n-1}$, a homogeneous PPP $\Phi(n)$ with intensity $\lambda$ is generated and then the nearest point in $\Phi(n)$ is selected as the next waypoint, i.e., $\boldsymbol{X}_{n}=\arg \min _{\boldsymbol{x} \in \Phi(n)}\left\|\boldsymbol{x}-\boldsymbol{X}_{n-1}\right\|$. The selection of velocity and pause time follow the traditional RWP mobility model. In particular, velocities $V_{n}$ are i.i.d. with distribution $P_{V}(\cdot)$. Pause times $S_{n}$ are also i.i.d. with distribution $P_{S}(\cdot)$.

Under the proposed model, different mobility patterns can be captured by choosing different $\lambda$ 's. Larger $\lambda$ statistically implies that the transition lengths $L$ are shorter. These $\lambda$ 's may be appropriate for mobile users walking and shopping in the city. In contrast, smaller $\lambda$ statistically implies that the transition lengths $L$ are longer. These $\lambda$ 's may be appropriate for driving users, particularly those on the highways. This intuitive result can also be observed in Fig. 1, which shows 4 sample traces of the proposed RWP mobility model.

\section{Stochastic PROPERTIES OF THE PROPOSED RWP MOBILITY MODEL}

In this section, we study the various stochastic properties of the proposed RWP mobility model. Stochastic properties of interests include transition length, transition time and spatial node distribution.

\section{A. Transition length}

We define transition length as the Euclidean distance between two successive waypoints. In the proposed model, the transition lengths can be described by a stochastic process
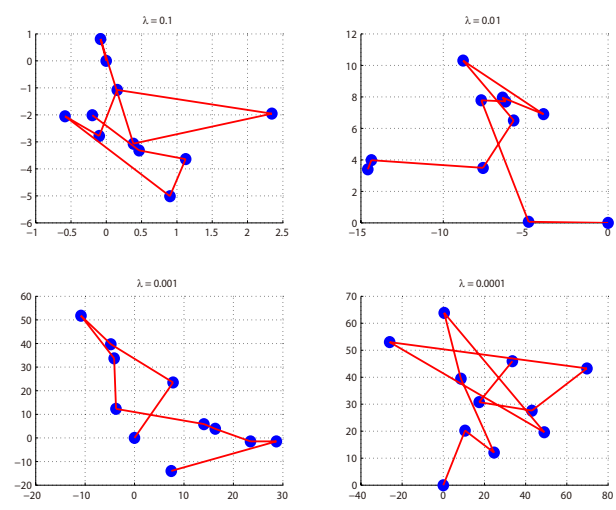

Fig. 1. Sample traces of the proposed RWP mobility model with $\lambda$ equals $10^{-1}, 10^{-2}, 10^{-3}$ and $10^{-4}$ respectively

$\left\{L_{n}\right\}_{n \in \mathbb{N}}$ where $L_{n}$ are independent and identically Rayleigh distributed with

$$
\mathbb{E}[L]=\frac{1}{2 \sqrt{\lambda}} .
$$

Note that the transition lengths are not i.i.d. in the traditional RWP mobility model. For example, a node currently located near the border of the finite domain tends to have a longer transition length while a node located around the centre of the finite domain statistically has a shorter transition length for the next movement period.

Nevertheless, the random waypoints $\boldsymbol{X}_{n}$ are i.i.d. in the traditional model, which is obvious since these waypoints are selected uniformly from a finite domain and independently over movement periods. This property forms the basis of the analysis of the traditional RWP mobility model (see, e.g., [4], [14]). In contrast, the waypoints in our proposed model are not i.i.d. but form a Markov process.

\section{B. Transition time}

We define transition time as the time a node spends during the movement between two successive waypoints. We denote by $T_{n}$ the transition time for the movement period $n$. Then $T=L / V$ where we omit the period index $n$ since $T_{n}$ are i.i.d.. Denote $\mathcal{V} \in \mathbb{R}$ as the range of the random velocity $V$. Given any velocity distribution $P_{V}(\cdot)$, the probability distribution of $T$ is given as follows.

Proposition 1. The cdf of the random transition time $T$ is given by

$$
P(T \leq t)=1-\int_{\mathcal{V}} \exp \left(-\lambda \pi v^{2} t^{2}\right) d P_{V}(v), t \geq 0 .
$$

As a specific application of Prop. 1, the following corollary gives closed form expressions for transition times under two types of velocity distributions.

Corollary 1. 1) If $V \equiv \nu$ where $\nu$ is a positive constant, the pdf of transition time $T$ is

$$
f_{T}(t)=2 \pi \lambda \nu^{2} t e^{-\lambda \pi \nu^{2} t^{2}}, t \geq 0 .
$$


2) If $V$ is uniformly distributed on $\left[v_{\min }, v_{\max }\right]$, the pdf of transition time $T$ is

$$
f_{T}(t)=\frac{g\left(v_{\min }\right)-g\left(v_{\max }\right)}{\left(v_{\max }-v_{\min }\right) t}, t \geq 0
$$

where $g(x)=x e^{-\lambda \pi t^{2} x^{2}}+\frac{1}{\lambda^{1 / 2} t} Q(\sqrt{2 \pi \lambda} t x)$ is nonincreasing, and $Q(x)=\frac{1}{\sqrt{2 \pi}} \int_{x}^{\infty} e^{-\frac{u^{2}}{2}} d u$.

We now derive the mean of transition time which will be used later. Instead of applying the cdf of $T$, we notice that

$$
E[T]=E\left[\frac{L}{V}\right]=E[L] E\left[\frac{1}{V}\right]=\frac{1}{2 \sqrt{\lambda}} \int_{\mathcal{V}} \frac{1}{v} d P_{V}(v)
$$

From (6), we can easily obtain that $E[T]=\frac{1}{2 \nu \sqrt{\lambda}}$ if $V \equiv \nu$.

\section{Spatial node distribution}

In this subsection, we study spatial node distribution. To this end, let $\boldsymbol{X}_{0}$ and $\boldsymbol{X}_{1}$ be two successive waypoints. Given $\boldsymbol{X}_{0}$, we are interested in the probability that the moving node resides in some measurable set $\mathcal{A}$ during the movement from $\boldsymbol{X}_{0}$ to $\boldsymbol{X}_{1}$. For brevity, we only give the spatial node distribution with the assumption that the mobile node does not have pause time.

Theorem 1. Assume that $S_{n} \equiv 0, \forall n$, and that $\boldsymbol{X}_{0}$ is at the origin. Then the spatial node distribution between $\boldsymbol{X}_{0}$ and $\boldsymbol{X}_{1}$ is characterized by the pdf $f(r, \theta)$ given by

$$
f(r, \theta)=\frac{\sqrt{\lambda}}{\pi r} \exp \left(-\lambda \pi r^{2}\right)
$$

Proof: See Appendix A.

The physical interpretation of $f(r, \theta)$ goes as follows. Let $d A(r, \theta)$ be a small area around the point $(r, \theta)$ under the polar coordinate. Then the probability $P(d A(r, \theta))$ that the moving node resides in some measurable set $\mathcal{A}$ during the movement from $\boldsymbol{X}_{0}$ to $\boldsymbol{X}_{1}$ is approximately given by

$$
P(d A(r, \theta)) \approx f(r, \theta) \cdot|d A(r, \theta)|
$$

where $|d A(r, \theta)|$ denotes the area of the set $d A(r, \theta)$. Also, as noted in the proof in Appendix A, $f(r, \theta)$ can be regarded as the ratio of the expected proportion of transition time in the set $d A(r, \theta)$ to the area $|d A(r, \theta)|$.

\section{Application to Cellular Networks: A HANDOVER STUDY}

In this section, we study the impact of mobility on handover rate using the proposed model. Handover rate is formally defined as follows.

Definition 1. The handover rate is defined as the expected number $E[N]$ of handovers during one movement period divided by the expected transition time. Mathematically, handover rate is given by $H=E[N] / E[T]$.

Handover rate is directly related to network signalling overhead. Clearly, handover rate is low in large cells. However, large cells impose higher requirement on other network resources such as transmit power and spectrum. Thus, analytic results on handover rate is useful for network dimensioning. For brevity, we only consider the scenario with constant velocity, i.e., $V \equiv \nu$.

\section{A. Hexagonal model}

In this subsection, we focus on hexagonal cellular networks. We assume the typical mobile user is located at the origin. Then the expected number of handovers during one movement period can be computed as

$$
E[N]=\sum_{n=1}^{\infty} n \int_{C_{n}} P(d A(r, \theta))
$$

where $P(d A(r, \theta))$ is the probability distribution of the waypoint density $f_{\boldsymbol{X}_{1}}(r, \theta)$ given in Lemma 1 and $C_{n}$ denotes the area covered by the $n$-th layer neighbouring cells. However, exact computation of $N$ by (9) is tedious. Thus, we propose the following approximation formula

$$
E[N]_{\mathrm{app}}=\sum_{n=1}^{\infty} n \int_{0}^{2 \pi} \int_{(2 n-1) R}^{(2 n+1) R} f_{\boldsymbol{X}_{1}}(r, \theta) r d r d \theta
$$

where $R=\sqrt{C / \pi}$ with $C$ being the hexagonal cell size, i.e., we approximate the $n$-th neighbouring layer by a ring with inner radius $(2 n-1) R$ and outer radius $(2 n+1) R$. This approximation captures the essence of (9) and yields Prop. 2

Proposition 2. Let $d$ be the side length of the hexagonal cell and $\lambda$ the mobility parameter. The expected number of handovers during one movement period $E[N]_{\text {app }}$ is given by

$$
E[N]_{\text {app }}=\sum_{n=0}^{\infty} \exp \left(-\frac{3 \sqrt{3}}{2}(2 n+1)^{2} \lambda d^{2}\right)
$$

and is bounded as $E[N]_{\text {app }}^{L} \leq E[N]_{\text {app }} \leq E[N]_{\text {app }}^{U}$ where

$$
\begin{aligned}
& E[N]_{\text {app }}^{L} \triangleq \sqrt{\frac{\pi}{6 \sqrt{3} \lambda d^{2}}} Q\left(\sqrt{3 \sqrt{3} \lambda d^{2}}\right) \\
& E[N]_{\text {app }}^{U} \triangleq \sqrt{\frac{\pi}{6 \sqrt{3} \lambda d^{2}}}\left(1-Q\left(\sqrt{3 \sqrt{3} \lambda d^{2}}\right)\right)
\end{aligned}
$$

Moreover, the difference $\triangle N_{\text {app }}\left(\lambda d^{2}\right)$ between the upper bound and lower bound is a strictly increasing function of $\lambda d^{2}$ and is within the range $(0,1)$. In particular, $\triangle N_{\text {app }}\left(\lambda d^{2}\right) \rightarrow$ 0 as $\lambda d^{2} \rightarrow \infty$, and $\triangle N_{\text {app }}\left(\lambda d^{2}\right) \rightarrow 1$ as $\lambda d^{2} \rightarrow 0$.

Using Prop. 2, the handover rate and the corresponding bounds readily follow by further dividing the expected transition time $E[T]$ which has been derived in Section III-B. In particular, when $d \rightarrow 0$,

$$
H_{\text {app }} \sim \sqrt{\frac{\pi}{6 \sqrt{3}}} \frac{\nu}{d}+o(1) .
$$

Though derivation by (9) is not tractable, the exact handover rate in hexagonal model can be obtained using generalized solution of Buffon's needle problem [15] as follows.

Proposition 3. Let $d$ be the side length of the hexagonal cell and $\lambda$ the mobility parameter. The expected number of 
handovers $E[N]$ during one movement period is

$$
E[N]=\frac{2 \sqrt{3}}{3 \pi d} \sqrt{\frac{1}{\lambda}}
$$

The handover rate $H=E[N] / E[T]$ is then given by

$$
H=\frac{4 \sqrt{3}}{3 \pi} \frac{\nu}{d}
$$

We remark that the insights obtained by either approximate or exact approach are the same. Note that the hexagonal cell size $s_{H}$ is given by $3 \sqrt{3} d^{2} / 2$ in hexagonal tiling. So (14) can be written as $H_{\text {app }} \sim \sqrt{\frac{\pi}{4}} \frac{\nu}{\sqrt{s_{H}}}$. Similarly, the exact method yields $H=\frac{4}{\pi} \sqrt{\frac{\sqrt{3}}{2}} \frac{\nu}{\sqrt{s_{H}}}$. Both results imply that handover rate is inversely proportional to the square root of cell size $s_{H}$.

\section{B. Poisson-Voronoi model}

In this subsection, we focus on cellular networks modeled by Poisson-Voronoi tessellation [10]. We first give a brief description of Poisson-Voronoi tessellation. Consider a locally finite set $\phi=\left\{\boldsymbol{x}_{i}\right\}$ of points $\boldsymbol{x}_{i} \in \mathbb{R}^{2}$, referred to as nuclei. The Voronoi cell $\mathcal{C}_{\boldsymbol{x}_{i}}(\phi)$ of point $\boldsymbol{x}_{i}$ is defined as

$$
\mathcal{C}_{\boldsymbol{x}_{i}}(\phi)=\left\{y \in \mathbb{R}^{2}:\left\|\boldsymbol{y}-\boldsymbol{x}_{i}\right\|_{2} \leq\left\|\boldsymbol{y}-\boldsymbol{x}_{j}\right\|_{2}, \forall x_{j} \in \phi\right\} .
$$

Let $\epsilon_{\boldsymbol{x}}$ be the Dirac measure at $\boldsymbol{x}$, i.e., for $A \in \mathbb{R}^{2}, \epsilon_{\boldsymbol{x}}(A)=1$ if $\boldsymbol{x} \in A$, and 0 otherwise. Then spatial point process $\Phi$ can be written as $\Phi=\sum_{i} \epsilon_{\boldsymbol{x}_{i}}$, and Poisson-Voronoi tessellation is defined as follows [16].

Definition 2. For a spatial Poisson point process $\Phi=\sum_{i} \epsilon_{\boldsymbol{x}_{i}}$ on $\mathbb{R}^{2}$, the union of the associated Voronoi cells, i.e., $\mathcal{V}(\Phi)=$ $\cup_{\boldsymbol{x}_{i} \in \Phi} \mathcal{C}_{\boldsymbol{x}_{i}}(\Phi)$, is called Poisson-Voronoi tessellation.

In cellular networks modeled by Poisson-Voronoi tessellation, the BSs are the nuclei located according to some PPP $\Phi$ in $\mathbb{R}^{2}$. Besides, each BS $\boldsymbol{x}_{i}$ serves mobile users which are located within its Voronoi cell $\mathcal{C}_{\boldsymbol{x}_{i}}(\Phi)$. The latter assumption is equivalent to the hypothesis of nearest BS association strategy. In the sequel, we also assume that PPP $\Phi$ modeling the deployment of the BSs is homogeneous and its intensity is denoted by $\mu$. Then we can prove Prop. 4 .

Proposition 4. Let $\mu$ be the intensity of the homogeneously PPP distributed BSs and $\lambda$ the mobility parameter. The expected number of handovers $E[N]$ during one movement period is

$$
E[N]=\frac{2}{\pi} \sqrt{\frac{\mu}{\lambda}}
$$

The handover rate $H=E[N] / E[T]$ is then given by

$$
H=\frac{4}{\pi} \nu \sqrt{\mu}
$$

The insight obtained here is consistent with those of regular hexagonal tessellation. In Poisson-Voronoi tessellation with the nuclei being homogeneous PPP $\Phi$ of intensity $\mu$, the expected value of the size $s_{H}$ of typical Voronoi cell is given by $s_{H}=E\left[\left|C_{o}(\Phi)\right|_{2}\right]=1 / \mu[7]$, where $C_{o}(\Phi)$ is the typical cell and $\left|C_{o}(\Phi)\right|_{2}$ denotes the area of $C_{o}(\Phi)$. So $H$ can be

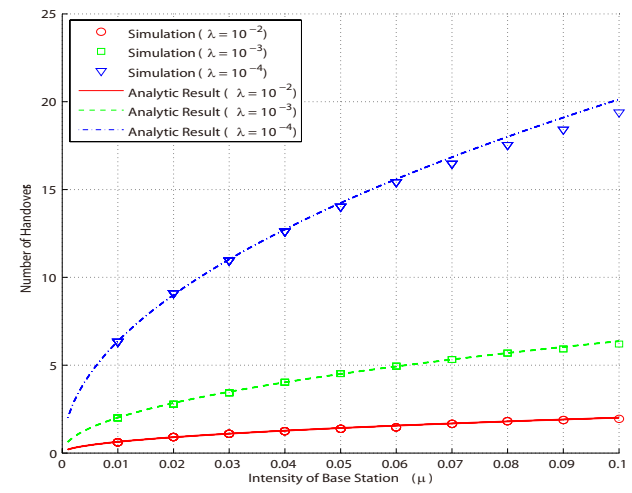

Fig. 2. Handover in Poisson-Voronoi model with velocity $\nu \equiv 1$.

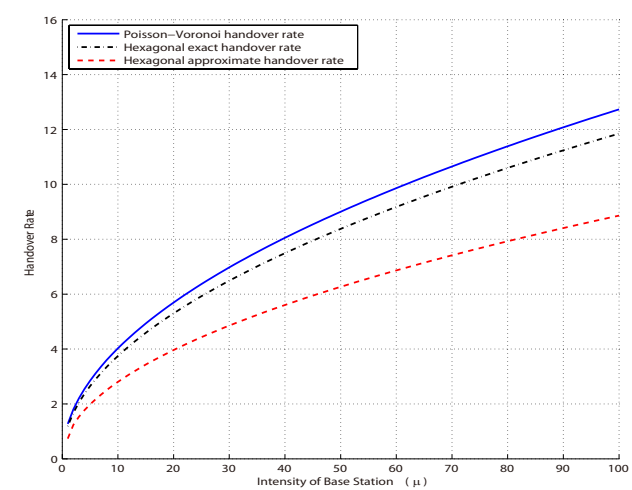

Fig. 3. Impact of BS density on handover rate: velocity $\nu \equiv 1$, BS intensity $\mu=1$ and mobility parameter $\lambda=1$.

written as $H=\frac{4}{\pi} \nu / \sqrt{s_{H}}$, which implies that handover rate is inversely proportional to the square root of cell size. This is consistent with the results in hexagonal model. Also, we note that the handover rate $H=\frac{4}{\pi} \nu \sqrt{\mu}$ has been derived in [17] using heuristic arguments rather than a formal proof.

Fig. 2 illustrates that the analytical result (17) matches the simulation result quite well. Also, we compare the handover rate of Poisson-Voronoi model, exact and approximate handover rate of hexagonal model in Fig. 3 as a function BS intensity. They all indicate that handover rate grows linearly with the square root of the BS intensity $\sqrt{\mu}$.

We finally evaluate the three types of analytic results on handover by simulating the proposed RWP mobility model using the real-world data of macro-BS deployment in a cellular network. The results are shown in Fig. 4. It can be seen that Poisson-Voronoi model is about as accurate as hexagonal model in predicting the number of handovers. Meanwhile, the approximate analytic result underestimates the number of handovers but more accurate in high $\lambda$ region.

\section{Conclunsions}

In this paper, we study mobility in cellular networks. To this end, we first propose a tractable RWP mobility model defined 


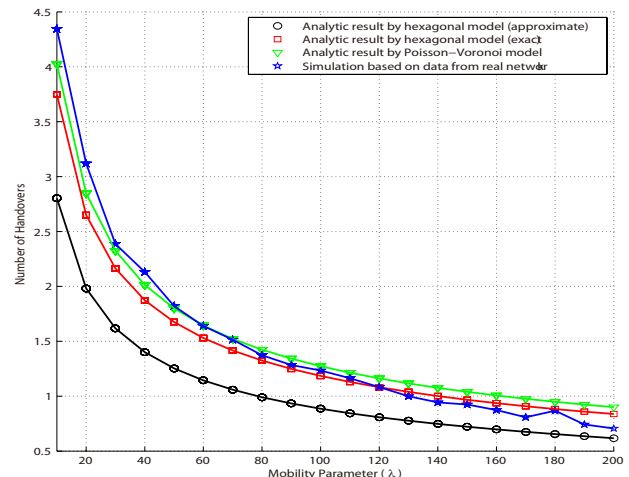

Fig. 4. Evaluation of handover by an actual macro-BS deployment with velocity $\nu \equiv 1$ and normalized BS intensity $\mu=400$.

on the entire plane. Key properties of the mobility model are carefully studied and (semi-)closed form expressions are obtained. Then we utilize this tractable mobility model to analyze the handover rate in cellular networks. The analysis is carried out for cellular networks under both hexagonal and Poisson-Voronoi models. The obtained analytic results are instrumental for mobility management in cellular networks.

\section{APPENDIX}

\section{A. Proof of Theorem 1}

We first derive the pdf of the random waypoint $\boldsymbol{X}_{1}=$ $\left(R_{1}, \Theta_{1}\right)$, which is summarized in Lemma 1.

Lemma 1. Given $\boldsymbol{X}_{0}$ is at the origin, the pdf of the random waypoint $\boldsymbol{X}_{1}=\left(R_{1}, \Theta_{1}\right)$ is given by

$$
f_{\boldsymbol{X}_{1}}(r, \theta)=\lambda \exp \left(-\lambda \pi r^{2}\right)
$$

Lemma 1 can be derived as follows:

$$
\begin{aligned}
f_{\boldsymbol{X}_{1}}(r, \theta) & =\lim _{\Delta r \rightarrow 0} \frac{P\left(R_{1} \leq r+\Delta r\right)-P\left(R_{1} \leq r\right)}{\int_{0}^{2 \pi} \int_{r}^{r+\Delta r} x d x d \phi} \\
& =\lim _{\Delta r \rightarrow 0} \frac{\exp \left(-\lambda \pi r^{2}\right)-\exp \left(-\lambda \pi(r+\Delta r)^{2}\right)}{2 \pi r \Delta r} \\
& =\lambda \exp \left(-\lambda_{i} \pi r^{2}\right)
\end{aligned}
$$

We next derive the pdf of the spatial node distribution. The main technique used is inspired by [18]. Consider a small set $d A$ located at $(r, \theta)$. Let $\vec{L}_{1}$ denotes the vector $\boldsymbol{X}_{1}-\boldsymbol{X}_{0}$ and $\left|\vec{L}_{1}\right|=L_{1}$. Then the pdf of the spatial node distribution can be interpreted as the ratio of the expected proportion of transition time in the set $d A$ to the area $|d A|$, i.e.,

$$
f(r, \theta)=\frac{E\left[\left|\vec{L}_{1} \cap d A\right| / V\right]}{E\left[L_{1} / V\right]|d A|}=\frac{E\left[\left|\vec{L}_{1} \cap d A\right|\right]}{E\left[L_{1}\right]|d A|}
$$

Note that

$$
\begin{aligned}
& \frac{E\left[\left|\vec{L}_{1} \cap d A\right|\right]}{|d A|}=\frac{\int_{r}^{\infty} f_{\boldsymbol{X}_{1}}(x, \theta) x d x d \theta \cdot \Delta l}{r \cdot d \theta \cdot \Delta l} \\
& =\frac{\int_{r}^{\infty} \lambda \exp \left(-\lambda \pi x^{2}\right) x d x}{r}=\frac{1}{2 \pi r} \exp \left(-\lambda \pi r^{2}\right)
\end{aligned}
$$

where $\Delta l$ denotes the length of the small intersection if $\vec{L}_{1}$ intersects $d A$, and we apply Lemma 1 in the second equality in (21). The first equality in (21) follows since

$$
E\left[\left|\vec{L}_{1} \cap d A\right|\right]=\Delta l \cdot \int_{r}^{\infty} f_{\boldsymbol{X}_{1}}(x, \theta) x d x d \theta
$$

and $|d A|=r \cdot d \theta \cdot \Delta l$. So

$$
f(r, \theta)=\frac{\exp \left(-\lambda \pi r^{2}\right)}{2 \pi r E\left[L_{1}\right]}=\frac{\sqrt{\lambda} \exp \left(-\lambda \pi r^{2}\right)}{\pi r}
$$

where we use the result that $E\left[L_{1}\right]=1 / 2 \sqrt{\lambda}$ in the last equality. This completes the proof.

\section{REFERENCES}

[1] T. Camp, J. Boleng, and V. Davies, "A survey of mobility models for ad hoc network research," Wireless Communications and Mobile Computing, vol. 2, no. 5, pp. 483-502, 2002.

[2] I. Akyildiz, J. Mcnair, J. Ho, H. Uzunalioglu, and W. Wang, "Mobility management in next-generation wireless systems," Proceedings of the IEEE, vol. 87, no. 8, pp. 1347-1384, August 1999.

[3] D. Johnson and D. Maltz, "Dynamic source routing in ad hoc wireless networks," Mobile Computing, pp. 153-181, 1996.

[4] C. Bettstetter, H. Hartenstein, and X. Pérez-Costa, "Stochastic properties of the random waypoint mobility model," Wireless Networks, vol. 10, no. 5, pp. 555-567, September 2004.

[5] P. J. Fleming, A. L. Stolyar, and B. Simon, "Closed-form expressions for other-cell interference in cellular cdma," Technical Report 116, Univ. of Colorado at Boulder, December 1997. [Online]. Available: http://ccm.ucdenver.edu/reports/

[6] T. X. Brown, "Cellular performance bounds via shotgun cellular systems," IEEE Journal on Selected Areas in Communications, vol. 18 no. 11 , pp. 2443 - 55, November 2000.

[7] D. Stoyan, W. Kendall, J. Mecke, and L. Ruschendorf, Stochastic geometry and its applications. Wiley New York, 1995, vol. 313.

[8] M. Haenggi, Stochastic Geometry for Wireless Networks. Cambridge University Publishers, 2012.

[9] M. Haenggi, J. Andrews, F. Baccelli, O. Dousse, and M. Franceschetti, "Stochastic geometry and random graphs for the analysis and design of wireless networks," IEEE Journal on Selected Areas in Communications, vol. 27, no. 7, pp. 1029-1046, September 2009.

[10] F. Baccelli and B. Blaszczyszyn, Stochastic Geometry and Wireless Networks, Part I: Theory. Now Publishers Inc, 2009.

[11] F. Baccelli and S. Zuyev, "Stochastic geometry models of mobile communication networks," Frontiers in Queueing, pp. 227-243, 1997.

[12] J. Andrews, F. Baccelli, and R. Ganti, "A tractable approach to coverage and rate in cellular networks," IEEE Transactions on Communications, vol. 59, no. 11, pp. 3122 -3134, November 2011.

[13] X. Lin, R. K. Ganti, P. J. Fleming, and J. G. Andrews, "Towards understanding the fundamentals of mobility in cellular networks," Technical Report, Univ. of Texas at Austin, March 2012. [Online]. Available: https://webspace.utexas.edu/xl3553/website/Mobility.pdf

[14] E. Hyytia, P. Lassila, and J. Virtamo, "Spatial node distribution of the random waypoint mobility model with applications," IEEE Transactions on Mobile Computing, pp. 680-694, June 2006.

[15] L. Schroeder, "Buffon's needle problem: An exciting application of many mathematical concepts," Mathematics Teacher, vol. 67, no. 2, pp. 183-186, 1974

[16] J. Møller, Lectures on random Voronoi tessellations. Springer-Verlag, 1994, vol. 87

[17] F. Baccelli and S. Zuyev, "Poisson-voronoi spanning trees with applications to the optimization of communication networks," Operations Research, pp. 619-631, 1999.

[18] C. Bettstetter, G. Resta, and P. Santi, "The node distribution of the random waypoint mobility model for wireless ad hoc networks," IEEE Transactions on Mobile Computing, pp. 257-269, September 2003. 